\title{
Comunicação
}

[Communication]

\section{Atividade inibitória do óleo essencial de orégano em fungos de importância médica e veterinária}

[Inhibitory activity of origanum essential oil against important fungus in veterinary]

\author{
M. B. Cleff ${ }^{1}$, A.R. Meinerz ${ }^{2}$, R.O. Faria ${ }^{2}$, M.O. Xavier ${ }^{1}$, R. Santin ${ }^{2}$, P.S. Nascente ${ }^{2}$, \\ M.R. Rodrigues ${ }^{3}$, M.C.A. Meireles ${ }^{1}$ \\ ${ }^{1}$ Faculdade Veterinária - UFPel - Pelotas, RS \\ ${ }^{2}$ Aluno de pós-graduação - FV-UFRGS - Porto Alegre, RS \\ ${ }^{3}$ Instituto de Química, UFPel - Pelotas, RS
}

\begin{abstract}
A crescente importância clínica atribuída às micoses em animais domésticos (Meinerz et al., 2007; Prestes et al., 2008), aliada às dificuldades representadas pelo tempo de administração, toxicidade e alto custo dos antifúngicos, tem impulsionado a realização de pesquisas na tentativa de se obter outras opções terapêuticas (Chami et al., 2004; Giordani et al., 2004). Nesse contexto, os extratos vegetais vêm ganhando espaço no cotidiano veterinário (Carmo et al., 2008; Prestes et al., 2008; Rusenova e Parvanov, 2009; Cleff et al., 2010). Assim, o estudo teve como objetivos avaliar a ação do óleo essencial do orégano (Origanum vulgare) em isolados fúngicos de importância na clínica veterinária, assim como analisar os constituintes químicos do óleo testado.
\end{abstract}

Utilizaram-se dois isolados humanos e 25 isolados clínicos de animais: Candida albicans $(\mathrm{n}=8)$; Sporothrix schenckii $(\mathrm{n}=7) ;$ Malassezia pachydermatis $(\mathrm{n}=8) ;$ A. flavus $(\mathrm{n}=2)$ e $A$. fumigatus $(\mathrm{n}=2)$. $\mathrm{O}$ óleo foi extraído por hidrodestilação em Clevenger e analisado por cromatografia em equipamento GC/FID (Schimadzu-17 A), nas seguintes condições: $40^{\circ} \mathrm{C}-2^{\circ} \mathrm{C} / \mathrm{min} ; 145^{\circ} \mathrm{C}-10^{\circ} \mathrm{C} / \mathrm{min} ; 280^{\circ} \mathrm{C}-10$ $\min ; \mathrm{Td}=280^{\circ} \mathrm{C} ;$ Tinj $=280^{\circ} \mathrm{C} ; \mathrm{T} \mathrm{col}=40^{\circ} \mathrm{C}$ e split $=1: 50$, sendo este comparado com os tempos de retenção de padrões terpênicos (Rodrigues et al., 2004).

A técnica de microdiluição em caldo foi a utilizada (NCCLSM27-A2 e M3). Os inóculos fúngicos foram ajustados em $1-5 \times 10^{6} \mathrm{UFC} / \mathrm{mL}$ para as leveduras e $S$. schenckii, e ajustados em $5 \times 10^{4} \mathrm{UFC} / \mathrm{mL}$ para Aspergillus. O óleo foi submetido a nove diluições $(2,0$ a $0,004 \%)$ em RPMI 1640 e adicionado de Tween $80(0,01 \%)$; para $M$. pachydermatis, utilizou-se Sabouraud líquido. As microplacas foram incubadas a $37^{\circ} \mathrm{C}$ por $48 \mathrm{~h}$ e por $72-96 \mathrm{~h}$ para os isolados de Aspergillus. A leitura da CIM correspondeu à menor concentração do óleo capaz de inibir o crescimento fúngico.

A análise cromatográfica identificou 34 constituintes, sendo 4-terpineol (41.17), timol (21.95), gamaterpinemo (5.91), alfaterpineol (4.98), carvacrol (4.71), betacariofileno (3.22) e metilcarvacrol (3.04), aqueles em maiores concentrações (Fig. 1). A literatura cita que fenóis, como carvacrol, timol, gamaterpeno e pcimeno, representam $70,2 \%$ a $98 \%$ dos compostos ativos do óleo de $O$. vulgare (Rodrigues et al., 2004).

Dentre os fungos estudados, M. pachydermatis, A. flavus e A. fumigatus apresentaram maior sensibilidade ao óleo de orégano (Tab. 1; Fig. 2), concordando com outros autores, que obtiveram CIM do óleo entre $0,06 \%$ e $0,25 \%$ para $M$. pachydermatis (Prestes et al., 2008; Rusenova e Parvanov, 2009), enquanto Carmo et al. (2008) observaram atividade do orégano antiAspergillus com CIM entre 20 e $80 \mu \mathrm{L} / \mathrm{mL}$.

Recebido em 27 de novembro de 2009

Aceito em 10 de setembro de 2010

E-mail: emebrum@bol.com.br 


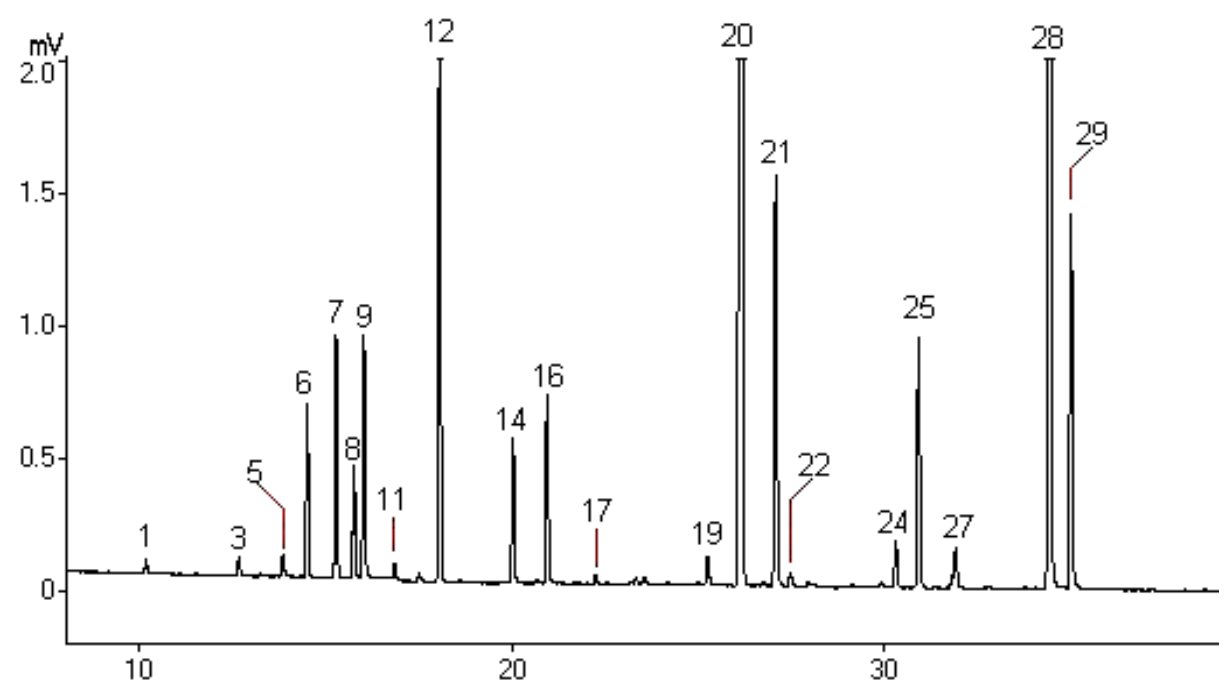

Figura 1. Cromatograma correspondente a amostra do óleo essencial do orégano, representando os principais constituintes, $\mathrm{p}=$ pico: $\alpha$-tujeno (p1), sabineno (p3), mirceno (p5), $\alpha$-felandreno (p6), $\alpha$ terpineno (p7), p-cimeno (p8), limoneno (p9), cis/trans $\beta$-cimeno (p11), gamaterpinemo (p12), terpinoleno (p14), linalol (p16), trans-p-mentenol (p17), borneol (p19), 4- terpineol (p20), $\alpha$-terpineol (p21), transpiperitol (p22), éter de metil timol (p24), éter de metilcarvacrol (p25), geraniol/nerol (p27), timol (p28) e carvacrol (p29). *Compostos identificados por comparação com o tempo de retenção dos padrões.

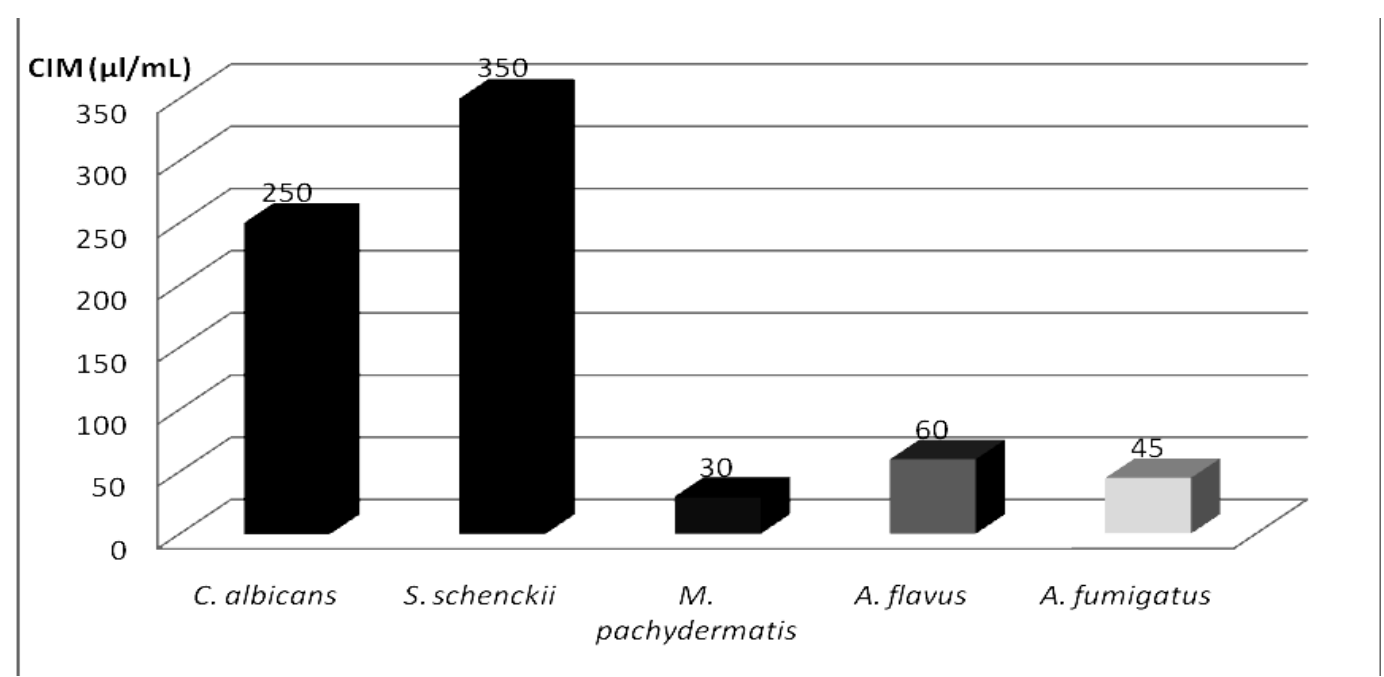

Figura 2. Representação gráfica dos valores médios de CIM do óleo essencial do Origanum vulgare (orégano) para diferentes espécies de fungos isolados de animais e do ambiente na região sul do Rio Grande do Sul.

A CIM para C. albicans e $S$. schenckii foi, em média, $0,3 \%$ (Tab. 1; Fig. 2), resultado satisfatório, já que estes agentes apresentam fatores de virulência e de resistência reconhecidos e são considerados de importância em saúde púbica (Chami et al., 2004; Meinerz et al., 2007; Cleff et al., 2010). Outros autores avaliaram compostos isolados do óleo em $C$. albicans e obtiveram CIM de $6.5 \mathrm{mM}$ para carvacrol e $12 \mathrm{mM}$ para eugenol (Chami et al., 2004). Em estudo prévio realizado por Cleff et al. (2010), ao utilizarem óleo de $O$. vulgare com alta concentração de 4-terpineol e proporções equilibradas de timol e carvacrol, demonstrou-se MIC de $2.72 \mu \mathrm{g} / \mathrm{mL}^{-1}$. 
Tabela 1. Valores de concentração inibitória mínima para o óleo essencial do Origanum vulgare (\%v/v) em espécies fúngicas isoladas de animais, humano e ambiente

\begin{tabular}{|c|c|c|c|c|c|c|c|c|c|c|}
\hline Isolados & 1 & 0,5 & 0,25 & 0,125 & 0,06 & 0,03 & 0,015 & 0,007 & 0,003 & 0,001 \\
\hline 01. C. albicans & - & - & - & + & + & + & + & + & + & + \\
\hline 02. C. albicans & - & - & - & - & + & + & + & + & + & + \\
\hline 03. C. albicans & - & - & - & + & + & + & + & + & + & + \\
\hline 04. C. albicans & - & - & - & - & + & + & + & + & + & + \\
\hline 05. C. albicans & - & - & - & - & + & + & + & + & + & + \\
\hline 06. C. albicans & - & - & - & + & + & + & + & + & + & + \\
\hline 07. C. albicans & - & - & + & + & + & + & + & + & + & + \\
\hline 08. C. albicans & - & - & - & + & + & + & + & + & + & + \\
\hline 09. S. schenckii & - & - & - & + & + & + & + & + & + & + \\
\hline 10. S. schenckii & - & - & + & + & + & + & + & + & + & + \\
\hline 11. S. schenckii & - & - & + & + & + & + & + & + & + & + \\
\hline 12. S. schenckii & - & - & - & + & + & + & + & + & + & + \\
\hline 13. S. schenckii & - & - & - & + & + & + & + & + & + & + \\
\hline 14. S. schenckii & - & - & + & + & + & + & + & + & + & + \\
\hline 15. S. schenckii & - & - & - & + & + & + & + & + & + & + \\
\hline 16. M. pachydermatis & - & - & - & - & - & - & + & + & + & + \\
\hline 17. M. pachydermatis & - & - & - & - & - & - & + & + & + & + \\
\hline 18. M. pachydermatis & - & - & - & - & - & - & - & + & + & + \\
\hline 19. M. pachydermatis & - & - & - & - & - & - & - & + & + & + \\
\hline 20. M. pachydermatis & - & - & - & - & - & - & + & + & + & + \\
\hline 21. M. pachydermatis & - & - & - & - & - & - & + & + & + & + \\
\hline 22. M. pachydermatis & - & - & - & - & - & - & - & + & + & + \\
\hline 23. M. pachydermatis & - & - & - & - & - & - & - & + & + & + \\
\hline 24. A. flavus & - & - & - & - & - & + & + & + & + & + \\
\hline 25. A. flavus & - & - & - & - & - & + & + & + & + & + \\
\hline 26. A. fumigatus & - & - & - & - & - & + & + & + & + & + \\
\hline 27. A. fumigatus & - & - & - & - & - & - & + & + & + & + \\
\hline
\end{tabular}

Sinal (-) representa inibição do crescimento fúngico, sendo considerada a CIM; sinal (+) representa crescimento fúngico. Números indicam a procedência do isolado: 1 a 6-mucosa vaginal de fêmeas caninas, 7- tegumento cutâneo de cão, 8- tegumento cutâneo de macaco; 9 a 13- esporotricose felina, 14 e 15- esporotricose humana, 16 a 23- meato acústico externo canino, 24 e 25 - ambiente, 26 e 27 - aspergilose pulmonar em pinguins.

Os valores de CIM podem ter sido influenciados pela razão molar dos compostos timol/carvacrol (4:1) associados à alta concentração de 4terpineol no óleo utilizado, já que estes têm sido descritos como responsáveis pela ação antimicrobiana do orégano (Lambert et al., 2001; Ultee et al., 2002; Chami et al., 2004). Estes compostos atuam induzindo deformações na membrana celular, alterando a permeabilidade. Além disso, é possível que núcleos aromáticos, contendo um grupo polar, possam fazer ligações de hidrogênio com os sítios ativos de enzimas microbianas alvo, o que favoreceria a atividade antimicrobiana (Lambert et al., 2001; Ultee et al., 2002).

Os resultados in vitro permitem concluir que o óleo do $O$. vulgare apresenta ação antifúngica, porém é importante realizar análises in vivo que confirmem a relação destes resultados, avançando na busca de alternativas terapêuticas aos antifúngicos.

Palavras-chave: fungos, óleo essencial, orégano 


\begin{abstract}
The in vitro activity of Origanum vulgare essential oil against fungal isolates was evaluated. A total of 27 clinical isolates were used, including: C. albicans, S. schenckii, M. pachydermatis, Aspergillus flavus, and A. fumigatus. Microdilution in broth technique (NCCLS M27-A2 and M-38) was used and susceptibility was expressed as Minimum Inhibitory Concentration (MIC). Essential oil was obtained by hydrodistillation in Clevenger and analyzed by gas chromatography, showing the presence of 4-terpineol, alpha-terpineol, 4-terpinene, thymol and carvacrol, as the main compounds. Origanum oil MIC for C. albicans varied from 125 to $500 \mu \mathrm{L} / \mathrm{mL}$; for $\mathrm{S}$. schenckii, from 250 to $500 \mu \mathrm{L} / \mathrm{mL}$; for M. pachydermatis, from 15 to $30 \mu \mathrm{L} / \mathrm{mL}$; and for Aspergillus, from 30 to $60 \mu \mathrm{L} / \mathrm{mL}$. Isolates sensitivity showed to the origanum oil stimulates the accomplishment of new studies, including in vivo tests, contributing to the search of alternative treatments to mycosis.
\end{abstract}

Keywords: fungal, essential oil, origanum

\section{REFERÊNCIAS BIBLIOGRÁFICAS}

CARMO, E.S.; LIMA, E.O.; SOUZA, E.L. The potential of origanum vulgare L. (Lamiaceae) essential oil in inhibiting the growth of some food-related Aspergillus species. Braz. J. Microbiol., v.39, p.362-367, 2008.

CHAMI, N.; CHAMI, F.; BENNIS, S. et al. Antifungal treatment with carvacrol and eugenol of oral candidiasis in immunosuppressd rats. Braz. J. Infec. Dis., v.8, p.217-226, 2004.

CLEFF, M.B.; MEINERZ, A.R.M.; XAVIER, M.O. et al. In vitro activity of Origanum vulgare essential oil against candida species. Braz. J. Microbiol., v.41, p.116-123, 2010.

GIORDANI, R.; REGLI, P.; KALOUSTIAN, J. et al. Antifungal effect of various essential oils against $C$. albicans. Potentiation of antifungal action of amphotericin B by essential oil from Thymus vulgaris. Phytot. Res., v.18, p.990-995, 2004.

LAMBERT, R.J.W.; SKANDAMIS, P.N.; COOTE, P.J. A study of the minimum inhibitory concentration and mode of action of oregano essencial oil, thymol and carvacrol. J. Appl. Microbiol., v.91, p.453-462, 2001.
MEINERZ, A.R.M.; CLEFF, M.B.; NASCENTE, P.S. et al. Esporotricose felina, micose de interesse em saúde pública. Rev. Bras. Med. Vet., v.29, p.174-176, 2007.

PRESTES, L.S.; SCHUCH, L.F.D.; MEIRELES, M.C.A. et al. Actividad de extractos de orégano y tomillo frente a microorganismos asociados con otitis externa. Rev. Cubana Plant. Medic., v.13, p.4-8, 2008.

RODRIGUES, M.R.A.; KRAUSE, L.C.; CARAMÃO, E.B. et al. Chemical composition and extraction yield of the extract of Origanum vulgare obtained from sub- and supercritical $\mathrm{CO}_{2}$. J. Agric. Food Chem., v.52, p.3042-3047, 2004.

RUSENOVA, N.; PARVANOV, P. Antimicrobial activities of twelve essential oils against microorganisms of veterinary importance. Trakia J. Sci., v.7, p.37-43, 2009.

ULTEE, A.; BENNINK, M.H.J.; MOEZELAAR, R. The phenolic hydroxyl group of carvacrol is essential for action against the food-borne pathogen Bacillus cereus. Appl. Environ. Microbiol., v.68, p.1561-1568, 2002. 